\title{
Tomographic reconstruction using incremental and NFFT methods
}

\author{
Camila de Lima ${ }^{a 1}$ and Elias Helou Salomão Neto ${ }^{a}$ \\ ${ }^{a}$ Institute of Mathematics and Computer Sciences - ICMC, \\ University of São Paulo, São Carlos, SP, Brazil. \\ Received on September 30, 2016 / Accepted on June 25, 2017
}

\begin{abstract}
Projection and backprojection operators are the numerically intensive part of iterative methods in tomographic reconstruction. Some alternatives based on interpolation over a regular grid on the Fourier space or on nonequispaced fast Fourier transforms were developed in order to alleviate the computational cost. Both approaches substantially speed up the computation of the iterations of classical algorithms, but the incremental methods, such as OSEM, RAMLA, SAEM, among others, cannot benefit from these techniques. In this paper, it is presented an efficient approach where the nonequispaced fast Fourier transform (NFFT) is used in each sub-iteration of the incremental method "ordered subsets for transmission tomography" (OSTR) to perform the most expensive calculations: the projection and backprojection. The proposed method is applied to synchrotron radiation tomography and the results show a good performance.
\end{abstract}

Keywords: Tomographic reconstruction, incremental methods, fast computation of (back)projection, OSTR, NFFT.

\section{Introduction}

The problem in tomography is to recover a function $f: \mathbb{R}^{2} \rightarrow \mathbb{R}$ from its arc length integrals along straight lines, defined by the Radon transform [10]. An analytical strategy to solve this problem is the filtered backprojection (FBP), a method based on the inverse Fourier transform (IFT) and the Fourier slice theorem, which consists in two steps: the filtering of data in the frequency domain and the backprojection of the filtered data. Despite the quality of reconstructed images, the computational cost involved to obtain an image of dimension $n^{2}$ is $O\left(n^{3}\right)$, which is concentrated in the backprojection.

\footnotetext{
${ }^{1}$ E-mail Corresponding Author: cdlima@usp.br.
} 
Considering that the data set provided by tomographs generates a finite number of Radon transform samples, the function $f$ to be reconstructed is discrete. Thus, the problem can be modeled by a system of linear equations. However, direct elimination methods cannot be applied to solve the system because of the huge data and image dimensions, ill-posedness of the matrix of the system combined with noisy and incomplete data, among others. In this way, iterative methods can be used, such as the algebraic reconstruction techniques (ART) [10].

In order to reduce the effects of noise in the resulting images, statistical models (maximum likelihood - ML) were developed for emission tomography initially [14], and the expectation maximization (EM) algorithm to solve the resulting optimization problem [15]. Even with improvements in reconstructed images when compared to FBP, the EM algorithm requires a lot of iterations to obtain acceptable images. As each iteration cost is $O\left(n^{3}\right)$, algorithms to achieve good results in fewer iterations are required.

The class of incremental algorithms was developed aiming to speed up iterative methods for tomographic reconstruction. Ordered subsets expectation maximization (OSEM) [9], row-action maximum likelihood algorithm (RAMLA) [3], string-averaging expectation-maximization (SAEM) [8], and ordered subsets for transmission tomography (OSTR) [5] are some examples. Such methods process the data into subsets in each iteration, which accelerates the convergence of the results.

On the other hand, some techniques have been developed in the literature seeking to decrease the computational cost involved in each iteration of iterative methods, such as the fast slant stack [2], the hierarchical decomposition algorithm [7], the technique based on Fourier in log-polar grids [1] and the nonequispaced fast Fourier transform (NFFT) [13], where the computation of projection/backprojection operator has a time complexity of $O\left(n^{2} \log n\right)$.

Thus, the aim of this study is to propose an approach that combines the incremental method OSTR and the fast computation of projection/ backprojection operators using NFFT in order to reconstruct tomographic images efficiently. The proposed method was applied to reconstruct an image of an apple seed, using data obtained from synchrotron x-ray micro/nano tomography, which is a tomographic modality of high resolution and generates a large amount of data. The obtained results of this implementation show a good performance of the proposed method. 


\section{Transmission Tomography}

The main modality of transmission tomography is based on x-rays. In this type of scan one wants information about the physical constitution of the study object. During the process, x-ray beams are emitted, which pass through the object in various directions and positions, and finally, these intensities are detected. It results in images of transversal sections of the object, obtained from radiation attenuation measurements [10].

This process can be modeled as line integrals along straight lines associated to each ray beam, known as projections. Thus, the tomographic reconstruction problem is to recover the image $f: \mathbb{R}^{2} \rightarrow \mathbb{R}$ from its projections at various angles, defined by Radon transform (RT):

$$
\mathcal{R}[f](\theta, t):=\int_{\mathbb{R}} f\left(t\left(\begin{array}{c}
\cos \theta \\
\operatorname{sen} \theta
\end{array}\right)+s\left(\begin{array}{c}
-\operatorname{sen} \theta \\
\cos \theta
\end{array}\right)\right) d s .
$$

An alternative to obtain the inverse of the the $\mathrm{RT}$ in order to reconstruct the image $f$ is the Fourier slice theorem. This theorem states that the Fourier transform of a projection with angle $\theta$ is equal to a slice with the same angle of the Fourier transform of the image. So, it is possible to reconstruct the image using the inverse Fourier transform. But only a finite number of samples is determined and the other samples are obtained by interpolation, which degrades the quality of images. Strategies with better performance are based on the solution of the discrete reconstruction problem, defined in the following section.

\subsection{Discrete Problem}

The reconstruction problem from projections can be fully-discretized by the following system of linear equations:

$$
R x=b
$$

where $R \in \mathbb{R}^{m \times n}$ is the discretized RT, $x$ is the desired image to be determined and $b \in \mathbb{R}^{m}$ contains the RT samples measured by the tomographic scan. The products $R x$ and $R^{T} b$ represents projection and backprojection operators, respectively.

The ill-posedness of the matrix of the system combined with noisy and incomplete data obtained greatly affect the quality of reconstructed images. The statistical model for transmission tomography is presented in order to diminish the effect of these kind of errors. 


\subsection{Statistical Model for Transmission Tomography}

The statistical model for transmission tomography based on the Poisson distribution [5] is given by:

$$
\min _{x \geqslant 0}-L(x):=\sum_{i=1}^{m} h_{i}\left((R x)_{i}\right) .
$$

where $h_{i}(l)=f_{i} e^{-l}+d_{i}-y_{i} \log \left(f_{i} e^{-l}+d_{i}\right)$, such that $f_{i}$ is the blank scan count, $d_{i}$ is the mean number of background events and $y_{i}$ represents transmission events detected in the $i$ th detector, and $(R x)_{i}=\sum_{j=1}^{n} r_{i j} x_{j}$ is the $i$ th projection. The following sections present the proposed approach to solve this problem.

\section{Proposed Aproach: OSTR and NFFT}

Solving problem (3) using incremental methods is not an innovation. In this paper, it is proposed a new approach to solve the cited problem using the NFFT technique that computes the projection and backprojection operators with complexity $O\left(n^{2} \log n\right)$ flops and accelerates each sub-iteration of the incremental method OSTR. The description of the proposed strategy is presented in the following sections.

\subsection{Ordered Subsets for Transmission Tomography}

The incremental method ordered subsets for transmission tomography (OSTR) presented in this paper is proposed by Erdoğan and Fessler [5]. In this method, the ordered subsets principle, first proposed by Hudson and Larkin in the OSEM method [9], is applied to the separable paraboloidal surrogates algorithm (SPS) [6].

The index set $\{1,2, \ldots, m\}$ is divided into $s$ subsets $U_{1}, U_{2}, \ldots, U_{s}$ such that $\bigcup_{i=1}^{s} U_{i}=\{1,2, \ldots, m\}$ and $U_{i} \cap U_{j}=\emptyset$ if $i \neq j$. The image is updated after processing the data in each subset. Thus, each iteration of this method is defined as passing through all subsets, using as starting point the reconstruction obtained by the previous sub-iteration.

The full form of the OSTR algorithm is presented below. Note that the calculation of projection is required in the precomputation of $\gamma_{i}$ in (4) and in $\hat{l}_{i}$, represented by (6) in each sub-iteration. The coefficients $d_{j}^{*}$ in precomputation (4) and $x^{(k, l)}$ shown in (8), in each sub-iteration, require the computation of the backprojection operator. 


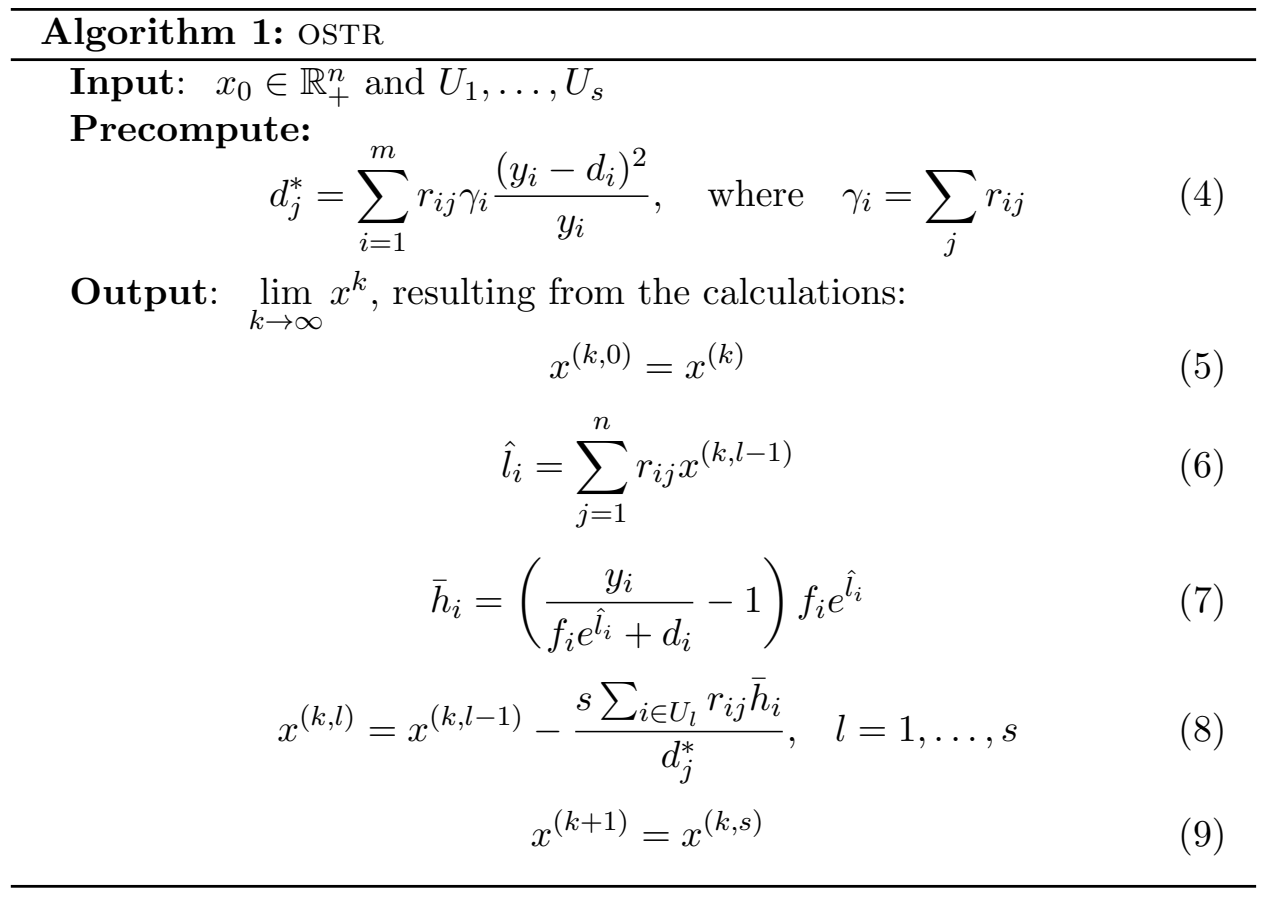

\subsection{Nonequispaced Fast Fourier Transform}

The nonequispaced fast Fourier transform (NFFT) generalizes the calculation of the fast Fourier transform (FFT) from equally spaced to arbitrary sampling points or spatial nodes [11]. In the image reconstruction, data are sampled regularly, and applying FFT, the data set remains in a regular grid, but using NFFT data can be obtained in an irregular grid. In this way, it is possible to use the Fourier slice theorem without explicit interpolation. Thereby, applying the two-dimensional NFFT followed by one-dimensional inverse FFTs (IFFTS), it is possible to obtain the projections of the image, and thus the RT.

The NFFT is used to compute the nonequispaced discrete Fourier transform (NDFT) in a fast and robust way [13]. Given the Fourier coefficients $\hat{f}_{k} \in \mathbb{C}, \boldsymbol{k} \in I_{N}$, the NDFT is defined as the evaluation of the corresponding trigonometric polynomial $f \in T_{N}$ at the set of $M$ arbitrary nodes $X$, given by the following sum:

$$
f(\boldsymbol{x}):=\sum_{\boldsymbol{k} \in I_{N}} \hat{f}_{\boldsymbol{k}} e^{-2 \pi i k \boldsymbol{x}}
$$

where $\boldsymbol{N}=\left(N_{t}\right)_{t=0, \ldots, d-1}$ is the multibandlimit and the possible frequencies $\boldsymbol{k} \in \mathbb{Z}^{d}$ are collect in the multi-index set: 


$$
I_{N}:=\left\{\boldsymbol{k}=\left(k_{t}\right)_{t=0, \ldots, d-1} \in \mathbb{Z}^{d}:-\frac{N_{t}}{2} \leq k_{t}<\frac{N_{t}}{2}, t=0, \ldots, d-1\right\} .
$$

This method is presented seeking to obtain a fast and efficient way to calculate the projections and backprojections to be evaluated in the subiterations of OSTR method, and achieve reasonable images in less time than existing strategies. In this paper, we use the $\mathrm{C}$ subroutine library named NFFT 3 [11], in the OSTR sub-iterations to compute the aforementioned operators.

\section{Results}

The computational tests were performed using data from an apple seed, which were obtained from synchrotron x-ray micro/nano tomography in the X-ray imaging (IMX) beamline of the Brazilian Synchrotron Light Laboratory (LNLS). Radon data was sampled in 512 angles between 0 and $\pi$ and the projection and reconstructed images has dimension of $2048 \times 2048$ pixels. From the data acquisition it was possible to obtain the sinogram, shown in Figure 1, and apply the proposed method to solve the statistical model of transmission tomography.

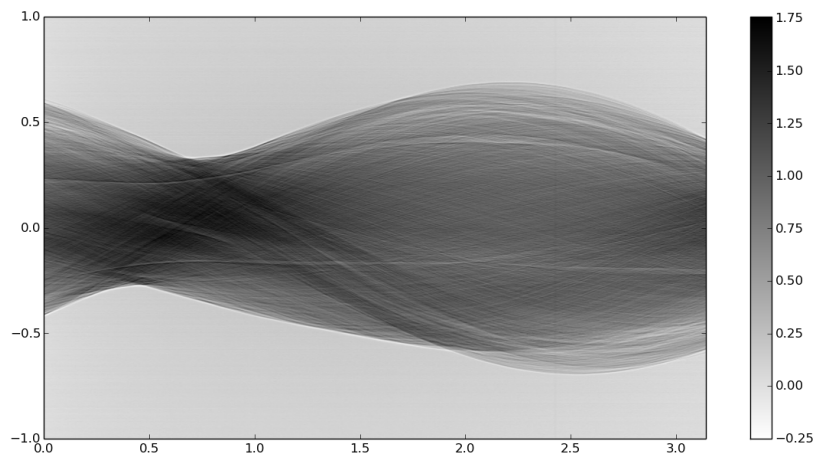

Figure 1 - RT of an apple seed.

We have considered algorithms using the full data set (equivalent to the SPS [6] without the ordered subsets principle), and data divided into 2, 4, 8 and 16 subsets in order to realize the results analysis, the complete data set data set. Figure 2 presents the images obtained in the first iteration of the mentioned configurations of the OSTR method, using NFFT in sub-iterations.

Figure 2 shows that the number of subsets is proportional to the amount of detail in the image obtained. However, the processing time of the iterations of the different OSTR method configurations increases with the number 
of subsets. The average time per iteration of the proposed method with full data set, 2, 4, 8 and 16 subsets, was 1.64, 2, 2.55, 4.03 and 5.96 seconds, respectively.

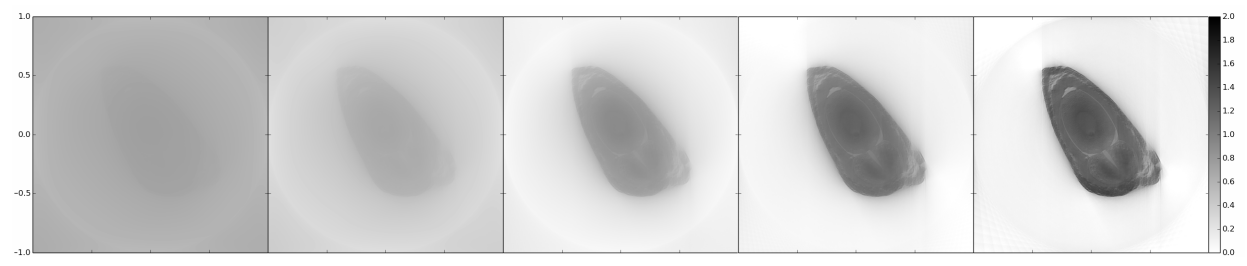

Figure 2 - First iteration of OSTR method using NFFT, with full data set, $2,4,8$ and 16 subsets, respectively.

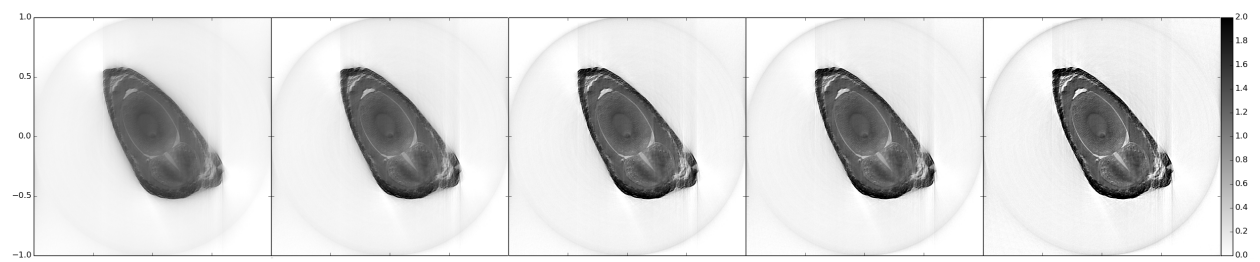

Figure 3 - Last iteration of the OSTR method using NFFT, with full data set, 2, 4, 8 and 16 subsets, respectively.

The configurations of the OSTR method using NFFT in the sub-iterations were compared in a period of 30 seconds. The reconstructed images obtained by the proposed method with full data set, 2, 4, 8 and 16 subsets in the end of the 30 seconds period are presented in Figure 3. Figure 4 shows the objective function values of the statistical model, achieved by the configurations in the same period of time.

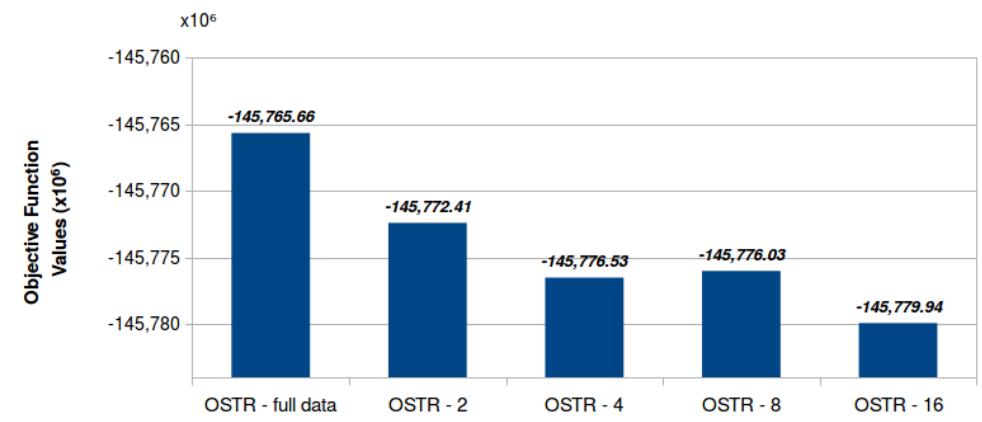

Figure 4 - Objective function values after 30 seconds of the execution of all configurations of the OSTR method using NFFT. 
The OSTR configurations proposed with full data set, 2, 4, 8 and 16 subsets take 19, 16, 12, 6 and 5 iterations, respectively, to achieve the presented results for the determined period of time. As presented in Figure 4, the highest value obtained for the objective function was $-145,765.664 \times 10^{6}$, from OSTR method with full data set. While the lowest value was $-145,779.939 \times$ $10^{6}$, found by OsTR method with 16 subsets. The images obtained by the OSTR method with full data set and 16 subsets after 30 seconds are shown in Figure 5.

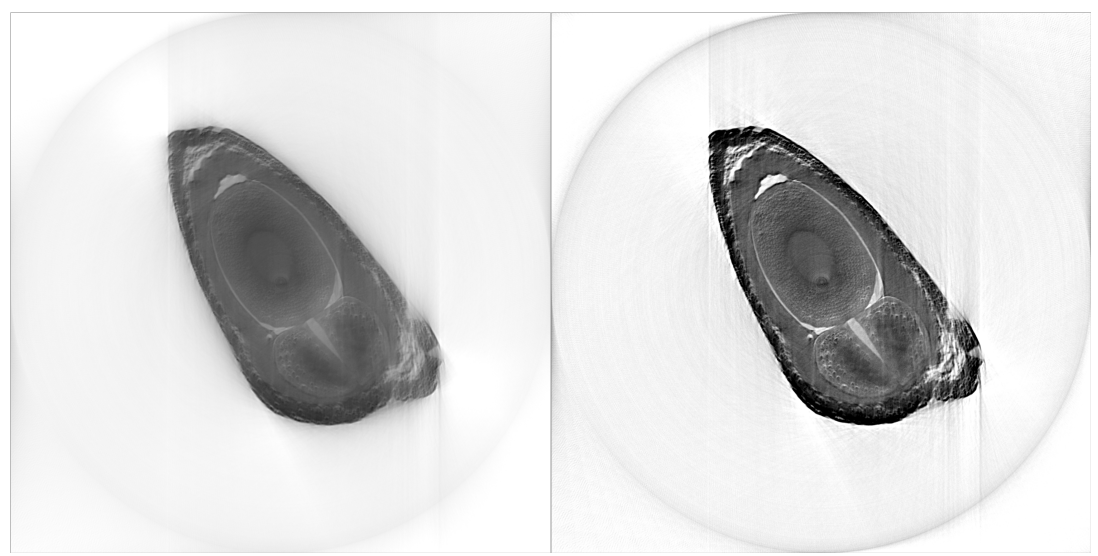

Figure 5 - Comparison between the worse (full data set) and the best (16 subsets) performance of OSTR using NFFT.

Figure 5 shows some details caused by ring artifacts in the image obtained by the final iteration of the proposed method. This problem can be solved using the generalized Tiratenko's algorithm, proposed by Miqueles et. al [12], which removes the rings of the image through the sinogram processing before the reconstruction.

\section{Final Considerations}

An efficient implementation of the OSTR method using in each subiteration the NFFT was proposed in this paper. The NFFT was used to compute the projection and backprojection operator and to reduce computational cost to $O\left(n^{2} \log n\right)$ flops. This method was applied to synchrotron light tomography and the results show a good performance, with images of quality in a efficient time.

The implementation of OSTR penalized method, using in each sub-iteration the NFFT, is one of the proposals for future work, as well as the implementation of OSTR with other techniques that perform the calculation 
of projection and backprojection operators with complexity $O\left(n^{2} \log n\right)$, such as the Fourier technique in a log-polar grid [1].

Acknowledgments. The authors would like to thank the PhD scholarship financed by FAPESP $(2013 / 16762-7)$, the projects financed by FAPESP (2013/16508-3 and 2013/07375-0) and CNPq (311476/2014-7), and to LNLS for allowing the testing in IMx lines.

\section{References}

[1] F. Andersson. Fast inversion of the radon transform using log-polar coordinates and partial back-projections. SIAM Journal on Applied Mathematics, 65(3):818837, 2005. doi:10.1137/S0036139903436005.

[2] A. Averbuch, R. R. Coifman, D. L. Donoho, M. Israeli and J. Waldén. Fast slant stack: A notion of Radon transform for data in a cartesian grid which is rapidly computible, algebraically exact, geometrically faithful and invertible. Technical Report, Statistics Department, Stanford University, 2001. URL: http://statweb.stanford.edu/ donoho/Reports/2001/FastSlantStack.pdf.

[3] J. Browne and A. R. De Pierro. A row-action alternative to the EM algorithm for maximizing likelihoods in emission tomography. IEEE Transactions on Medical Imaging, 15(5):687699, 1996. doi:10.1109/42.538946.

[4] A. R. De Pierro and M. E. B. Yamagishi. Fast EM-like methods for maximum a posteriori estimates in emission tomography. IEEE Transactions on Medical Imaging, 20(4):280288, 2001. doi:10.1109/42.921477.

[5] H. Erdoğan and J. A. Fessler. Ordered subsets algorithms for transmission tomography. Physics in medicine and biology, 44(11):2835-2851, 1999. doi:10.1088/0031-9155/44/11/311.

[6] H. Erdoğan and J. A. Fessler. Accelerated monotonic algorithms for transmission tomography. Proceedings IEEE International Conference on Image Processing, 2:680-684, 1998. doi:10.1109/ICIP.1998.723620.

[7] A. George and Y. Bresler. Fast tomographic reconstruction via rotationbased hierarchical backprojection. SIAM Journal on Applied Mathematics, 68(2):574597, 2007. doi:10.1137/060668614. 
[8] E. S. Helou, Y. Censor, T. Chen, I. Chern, A. R. De Pierro, M. Jiang and H. H. Lu. String-averaging expectation-maximization for maximum likelihood estimation in emission tomography. Inverse Problems, 30:120, 2014. doi:10.1088/0266-5611/30/5/055003.

[9] H. M. Hudson and R. S. Larkin. Accelerated image reconstruction using ordered subsets of projection data. IEEE Transactions on Medical Imaging, 13(4):601609, 1994. doi:10.1109/42.363108.

[10] A. C. Kak and M. Slaney. Principles of Computerized Tomographic Imaging. IEEE press, 1988.

[11] J. Keiner, S. Kunis and D. Potts. Using NFFT 3 - a software library for various nonequispaced fast Fourier transforms. ACM Transactions on Mathematical Software, 36(19): 1-30, 2009. doi:10.1145/1555386.1555388.

[12] E. X. Miqueles, J. Rinkel, F. O’Dowd and J. S. V. Bermúdez. Generalized Titarenko's algorithm for ring artefacts reduction. Journal of Synchrotron Radiation, 21: 1333-1346, 2014. doi:10.1107/S1600577514016919.

[13] D. Potts and G. Steidl. New Fourier reconstruction algorithms for computarized tomography. In A. Aldroubi, A. Laine e M. Unser Proceedings of SPIE: Wavelet Applications in Signal and Image Processing VIII, 4119:13-23, 2000. doi:10.1117/12.408606

[14] L. A. Shepp and Y. Vardi. Maximum likelihood reconstruction for emission tomography. IEEE Transactions on Medical Imaging, 2: 113122, 1982. doi:10.1109/TMI.1982.4307558.

[15] Y. Vardi, L. A. Shepp and L. Kaufman, A statistical model for positron emission tomography. Journal of the American Statistical Association. 80(389): 8-20, 1985. URL: http://www.jstor.org/pss/2288030. 